\title{
Investigación como estrategia pedagógica para fortalecer la comprensión lectora estudiantil ${ }^{1}$
}

\section{Research as a pedagogical strategy to strengthen student reading comprehension}

DOI: http://dx.doi.org/10.17981/cultedusoc.9.3.2018.105

Artículo de investigación. Fecha de recepción: 15/06/2018. Fecha de aceptación: 27/11/2018

Ana Elena Ramos-Diaz²;

María Melchora Meza-Meriño; Edith Cecilia Melendez-Carrillo; Hernando Jesús Meriño-Salazar y Alby Rosa Ramos-Berdugo ${ }^{3}$ Institución Educativa Escuela Urbana Mixta las Flores (Colombia)

ana_rey05@hotmail.com

Para citar este artículo:

Ramos-Diaz, A., Meza-Meriño, M., Melendez-Carrillo, E., Meriño-Salazar, H. y Ramos-Berdugo, A. (2018). Investigación como estrategia pedagógica para fortalecer la comprensión lectora estudiantil. Cultura. Educación y Sociedad 9(3), 885-892. DOI: http://dx.doi. org/10.17981/cultedusoc.9.3.2018.105

\section{Resumen}

La comprensión lectora es una competencia que desarrollan los estudiantes desde la educación básica, donde los docentes ayudan a crear estrategias pedagógicas que favorezcan a mojar las Competencias investigativas en los niños y niñas. El estudio tuvo como objetivo fortalecer la comprensión lectora estudiantil bajo la investigación como estrategia pedagógica. El estudio es de tipo cualitativa, descriptiva. Las técnicas de recolección la observación participante registrada en diarios de campo. Los participantes fueron los que presentaban mayor dificultad en compresión lectora, información suministrada de los resultados evaluativos de los periodos académicos, la unidad de análisis fue de cuarenta y dos (42) estudiantes de educación básica primaria del grado cuarto (4) de la institución educativa san Antonio sede escuela urbana mixta las flores. Los resultados arrojaron que las nuevas estrategias de investigación que implementaron los maestros ayudaron a desarrollar y mejorar la compresión lectora de los estudiantes.

Palabras clave: investigación como estrategia pedagógica, comprensión lectora, lectura.

\section{Abstract}

Reading comprehension is a competence that students develop from basic education, where teachers help create pedagogical strategies that favor wetting the investigative skills of children. The objective of the study was that research as a pedagogical strategy (IEP) help strengthen student reading comprehension. It was guided by the methodological guidelines of the IEP, using techniques such as participant observation and the field diary, participants were chosen students who had greater difficulty in reading comprehension so the unit of analysis was formed by forty-two (42) students of primary basic education grades (3) to (4) of the San Antonio educational institution headquarters mixed urban school flowers. The results showed that the new research strategies implemented by the teachers helped to develop and improve the reading comprehension of the students.

Keywords: research as pedagogical strategy, reading comprehension, reading.

1 Este artículo ha sido derivado del Programa de Fortalecimiento de la Cultura Ciudadana y Democrática CT+I a través de la IEP apoyada en TIC en el Departamento de Magdalena: CICLON

2Líder del grupo de investigación: Grupo de investigación "Los Búhos de la lectura".

3 Docentes de la Escuela Urbana Mixta las Flores y miembros del Grupo de investigación "Los Búhos de la lectura".

- The author; licensee Universidad de la Costa - CUC.

Cultura, Educación y Sociedad vol. 9 no. 3, pp. 885-892. Diciembre, 2018

Barranquilla. ISSN 2389-7724 Online 


\section{Introducción}

La educación a través de su evolución ha traído consigo el diseño, e implementación de nuevas estrategias pedagógicas, logrando extender la invitación a los maestros y maestras a la puesta en marcha de las mismas al interior del aula de clases, así mismo medir su impacto con el fin de direccionar la enseñanza propendiendo a la calidad educativa, para el desarrollo máximo de capacidades en los estudiantes.

Como la compresión lectora una de las competencias básicas que el estudiante adquiere desde básica primaria, estas competencias no solo son leer, ya que enmarca el hecho de brindarle un sentido al texto, las estrategias pedagógicas pueden ayudar a mejorar dicha habilidad entendiendo que son un conjunto de actividades que se realizan en un orden lógico y coherente en función del cumplimiento de objetivos. Es decir, es una planificación que contiene métodos o acciones que permiten al estudiante alcanzar los logros propuestos, mejorar su aprendizaje y facilitar su crecimiento personal (Picardo, Balmore, y Escobar, 2004).

Considerar la investigación como estrategia pedagógica en el fortalecimiento de la comprensión lectora implica recrear un espacio de inclusión, donde se respete y se viva la diversidad haciendo evidentes los canales de la creatividad del docente y el estudiante, para convertir las preguntas de los grupos de aprendizaje colaborativo conformados por niños, niñas y adolescentes en una investigación, llevándolos a tomar un rol activo en el proceso de aprendizaje mediante la construcción de su propia conocimiento, potenciando así, las habilidades y destrezas de los estudiantes, convirtiendo la educación en un vehículo de capacitación, de oportunidades y de recreación (Camargo, 2015).

Mejía y Manjarrez, (2010), afirman que la IEP, es una metodología encaminada a fomentar el interés en la población estudiantil por la investigación científica y tecnológica, donde el maestro acompaña a sus educan- dos en la búsqueda del saber a través de una acción dialógica que incluye el texto, la disciplina y el método científico. "permite llevar la investigación a la esfera educativa y pedagógica, que además genera una reelaboración del campo de éstas, así como de los mecanismos prácticos y de acción que las han constituido".

Por otro lado, la investigación es un proceso sistemático, organizado y objetivo, cuyo propósito es responder a una pregunta o hipótesis, aportando al aumento del conocimiento sobre un tema desconocido o poco estudiado. Este proceso se orienta a la obtención de nuevo conocimiento, necesario para el avance de la ciencia y tecnología en sus diversas aplicaciones (Hernández, Fernández y Baptista, 2010).

En resumen, el docente desde la IEP parte de la pregunta de sentido común de los estudiantes, la re direcciona a la perspectiva de la educación, reconociendo la existencia de saberes comunes o elaborados, asociando los conocimientos disciplinares con las experiencias socioculturales, promoviendo así la apropiación practica desde el pensamiento crítico buscando transformar contextos, culturas, epistemologías, y redirigir sus escenarios de poder para construir subjetividades y ciudadanías (Mariño, 2010).

Ramírez (2018), manifiesta que las tendencias emergentes, sirven como herramienta de gestión para la sociedad del conocimiento, lo cual puede ser aplicado al contexto educativo para generar transformaciones en la convivencia; facilitando nuevos estilos administrativos y operativos de labor institucional para una generación de valor agregado, enfoque sistémico humanizante hacia lo estratégico - dinamizador, inclusión de la ciencia y tecnología, capacidad de internacionalización y asociatividad; convirtiendose en factores claves de competitividad.

Desde lo educativo, es posible reflexionar acerca de la importancia del desarrollo e implementación de estrategias pedagógicas que busquen la potencialización de las habi- 
lidades y competencias de los estudiantes, que permitan la generación de conocimientos y aprendizajes significativos. Entre ellas es posible destacar la comprensión lectora, que se define como la reflexión y análisis obtenido a partir de la revisión de textos (Romero, Escorihuela y Ramos2009).

En los procesos de comprensión lectora se involucran el pensamiento y el lenguaje, de manera interrelacionada. Goodman, (1982) establece que hay un único proceso de lectura, el cual es aplicable a todas y cada una de las lenguas existentes. Manzano (2000), por su parte, considera a la lectura como aquella capacidad intelectual que se genera en el individuo al analizar, crear y descubrir los aspectos más profundos en términos de sensibilidad.

Desde ese punto de vista, es posible considerar la lectura como un proceso auto dirigido por un individuo que identifica de un texto el significado expresado por un escritor, y que incluye una serie de elementos que se configuran en múltiples estrategias en la solución de dificultades presentes al momento de leer (Secretaria de Educación Pública, 2001).

La lectura no es un trabajo fácil, este es una actividad ardua, ya que se necesita el desarrollo de habilidades lectoras y las competencias necesarias para alcanzar buenos niveles de comprensión (Gutiérrez, y Pérez, 2012; Restrepo, Gutiérrez, Caro y Moreno 2015).

En los procesos de aprendizaje en la lectura y el ejercicio mismo de leer, algunos autores identifican diferencias marcadas. Entre ellas es posible considerar que la escuela imparte la enseñanza de la lectura, al reconocer aspectos relacionados con las representaciones escritas en un sentido meramente operacional. Sin embargo, enseñar a leer no necesariamente significa comprender el sentido que el autor desea transmitir en sus escritos (Gómez, et al., 2015).

$\mathrm{Al}$ ser un proceso útil para el desarrollo de todas las áreas curriculares en los espacios académicos, la lectura evidencia su carácter transversal, generando efectos en el desarrollo y aprendizaje de tales áreas en sentido positivo o negativo (Fuchs, 2003). Resultados de investigaciones empíricas demuestran que quienes manifiestan mejores destrezas en comprensión lectora, suelen emplearlas eficientemente en la comprensión de los contenidos escolares, mientras que aquellos que poseen dificultades, tendrán más dificultad en los procesos de aprendizaje de los contenidos académicos (Paris, Lipson y Wixson 1983), lo cual los hace incapaces de recuperar los conocimientos previos asociados a lo leído (Marr y Gormley, 1982).

Según Franco, Blanco y Cortes, (2013) el proceso de lectoescritura debe contemplar tres aspectos fundamentales; en primer lugar; la comprensión, que indica la forma en la que las personas interpretan y analizan el lenguaje oral y escrito, a su vez esta categoría implica habilidades como percepción del habla, conocimiento del léxico, la manera como se analiza la estructura gramatical de las oraciones y el discurso que en un texto refiere a la formulación y evaluación de conversaciones o textos extensos. En segundo lugar; la producción del habla, que indica el modo de producción de lenguaje, donde la producción busca conocer cómo se convierten los conceptos a su forma lingüística es más difícil. Y, en tercer lugar; el proceso de adquisición que indica cómo se aprende una lengua.

En ese sentido Figueroa y Carrascal, (2016). Plantean que los procesos de lectoescritura son una necesidad actual, sin embargo, son las habilidades lingüísticas que presentan más falencias a nivel de educación básica y media en el país, lo cual se observa en los resultados de las pruebas de estado para medir el nivel educativo, donde el índice de desarrollo de pensamiento crítico de los estudiantes en las pruebas nacionales e internacionales, es muy bajo, sin embargo se ha observado que la educación a nivel rural, es muy poca, por lo cual los niveles de lectura y escritura son muy pobres. 
Por otro lado, el entorno educativo, es un sistema organizado que permite un nivel de interacción entre estudiantes, docentes y cuerpo administrativo, sin embargo, desde los cursos iniciales de básica primaria, se puede observar un nivel un nivel de comunicación primitivo como señas, gestos y grafos, que posibilita reconocer al otro a través de la escucha atenta junto a la percepción visual, encontrando la información necesaria para motivarlo a aprender el sistema estructurado de la lengua escrita y hablada, en ese sentido es necesario estimular el aprendizaje de la lectura y escritura en los primeros años, puesto que la curiosidad por desarrollarlos favorece el proceso de enseñanza, para esto utilizar la mediación de las tecnologías de la información puede ser una estrategia didáctica que permita la adquisición de habilidades en lectura y escritura (Franco, 2010; Marín, Niebles, Sarmiento, y Balvuena, 2017).

\section{Metodología}

\section{Diseño}

La investigación es de tipo cualitativa, de alcance descriptivo, basado en las trayectorias de indagación propia de la IEP que permiten el fomento la participación de los maestros y estudiantes a través del trabajo cooperativo, individual y el intercambio de saberes previos, así mismo, de la indagación, observación, análisis y evaluación de su entorno.

\section{Participantes}

La unidad de análisis estuvo conformada por cuarenta y dos (42) estudiantes del grado cuarto de primaria, con edades que oscilaron entre los ocho (8) y los trece (13) años de la Institución Educativa Departamental San Antonio, sede Las Flores, del municipio de Cerro de San Antonio, Magdalena.

\section{Técnicas e instrumentos}

En el estudio realizado se seleccionaron técnicas de recolección como la observación participante esta se distingue como la técnica que permanece en contacto con las personas observadas, como instrumento de recolección de información se utilizó el diario de campo consignando cada experiencia o hecho interpretando analizando después los resultados.

\section{Procedimientos}

El proceso de investigación estuvo enmarcado en 5 momentos los cuales ayudaron al diseño de las estrategias que fortalecieran la compresión lectora y fomentar la investigación desde un pensamiento crítico, se presentan a continuación los siguientes momentos:

- Momento (1): selección y análisis de la población sujeto de estudio.

- Momento (2): integración de la temática mediante la IEP al aula de clases, se integraron grupos estudiantiles para seleccionar problemáticas relacionadas con la compresión lectora. Se reunieron los estudiantes y con lluvia de ideas se plantearon preguntas problemas acompañados por el docente realizaron el proceso de indagación mediante herramientas virtuales teóricas conociendo nuevas estrategias para implementar.

- Momento (3): socialización de los resultados de las indagaciones de estudiantes en una feria institucional dirigida por los docentes.

- Momento (4): análisis de los resultados e implementación de la estrategia a través de la observación y diario de campo.

- Momento (5): propagación y divulgación de los resultados. 


\section{Resultados}

En la realización del proceso investigativo se logró observar que el grado cuarto de básica primaria hay estudiantes que se muestran con dificultades en compresión lectora, entre los más evidentes se resaltan: baja comprensión de textos cortos, identificación de los tipos de lecturas de acuerdo a gustos, pobreza de vocabulario, poco dominio en la resolución de problemas desencadenado de textos de análisis de menor complejidad.

Lo anterior permitió el diseño de las actividades a continuación (Ver Tabla 1).
Posterior al desarrollo de actividades para fortalecer los procesos de comprensión lectora los estudiantes aprenden nuevas y novedosas estrategias de lectura, diferencias tipos de textos, comprenden párrafos que requieren de análisis, destacando de los contenidos la posibilidad de escoger lo que ellos quisieran leer, identificando competencias en cada uno de los participantes, de la mano con la motivación, participación y preferencias de cierto tipos de lecturas.

TABLA 1

Sistematización de estrategias ejecutadas en el proyecto

\begin{tabular}{|c|c|c|}
\hline Estrategia & Descripción & Resultado \\
\hline La lectoescritura & $\begin{array}{l}\text { - Concursos sobre historias escritas por los estudiantes a } \\
\text { partir de sus vivencias cotidianas. } \\
\text { - Talleres de formación sobre la lectura y el uso de las TIC }\end{array}$ & $\begin{array}{l}\text { Se logró fomentar y formar } \\
\text { a los estudiantes desde la } \\
\text { sana competencia. }\end{array}$ \\
\hline Los formadores & $\begin{array}{l}\text { Se seleccionó un grupo de estudiantes de los grados más } \\
\text { avanzados para que hicieran las veces de tutores de los } \\
\text { más pequeños e intercambiar experiencias lectoras. }\end{array}$ & $\begin{array}{l}\text { Se logró la integración de } \\
\text { la población beneficiada } \\
\text { con la comunidad } \\
\text { estudiantil ayudando en } \\
\text { el avance significativo } \\
\text { de los estudiantes con } \\
\text { dificultades. }\end{array}$ \\
\hline Comunicándonos & $\begin{array}{l}\text {-Diseño de un periódico mural para compartir sus } \\
\text { historias. } \\
\text {-Elaboración de un blog que registre cada momento del } \\
\text { proyecto a ejecutar. } \\
\text {-Elaboración de una página en Facebook que junto al } \\
\text { blog se convertirán en herramientas de divulgación del } \\
\text { proyecto. }\end{array}$ & $\begin{array}{l}\text { Con la ayuda de los } \\
\text { maestros líderes se logró } \\
\text { que los participantes } \\
\text { a través de dichas } \\
\text { estrategias se cumpliera el } \\
\text { objetivo. }\end{array}$ \\
\hline $\begin{array}{l}\text { Jornada de } \\
\text { actividades / } \\
\text { Espacios para } \\
\text { leer }\end{array}$ & -Creación de historias a partir de una imagen. & $\begin{array}{l}\text { Se logró obtener mejor } \\
\text { relato sobre la imagen } \\
\text { que se observaba y que los } \\
\text { estudiantes hablaran sobre }\end{array}$ \\
\hline $\begin{array}{l}\text { Historias en } \\
\text { línea }\end{array}$ & $\begin{array}{l}\text { A través de la plataforma YouTube, se buscaron cuentos } \\
\text { y canciones historias, una vez observadas al final ellos } \\
\text { explicaron las partes más representativas de lo visto. }\end{array}$ & $\begin{array}{l}\text { el tema. } \\
\text { Gracias a la utilización de } \\
\text { estas estrategias se pudo }\end{array}$ \\
\hline $\begin{array}{l}\text { Descubriendo las } \\
\text { palabras }\end{array}$ & $\begin{array}{l}\text { Se les presentaron textos sobre situaciones cotidianas, } \\
\text { ellos identificaron palabras claves dentro de ellos. }\end{array}$ & $\begin{array}{l}\text { captar la atención ya que } \\
\text { hace parte de la nueva era. }\end{array}$ \\
\hline
\end{tabular}

Fuente: elaboración propia, (2018) 


\section{Discusión}

La Lectura es una herramienta fundamental de trasformación social, razón por la cual el presente proyecto que buscaba fortalecer la comprensión lectora en los estudiantes, tuvo en cuenta los bajo niveles de rendimiento de los estudiantes objetos de estudio y con ellos implementar las actividades.

Si bien es cierto que los estudiantes participaron activamente en el proceso se deben seguir diseñando y llevar a la práctica diversas metodologías para que se logre de la lectura un espacio de reflexión y aprendizaje constante.

Al ser el aprendizaje de la lectura y la escritura un aspecto tan complejo y determinante en la formación de seres humanos integrales, se requiere la creación de ambientes de aprendizaje lúdicos pedagógicos que despierten interés en los niños y niñas evidenciando la necesidad de fortalecer dichos procesos del grado cuarto de la Institución Educativa Departamental San Antonio sede Las Flores.

A través de la investigación como estrategia pedagógica se logró implementar estrategias innovadoras que permitieron la transformación de la práctica educativa, generando capacidades que anteriormente no eran desarrolladas con la educación tradicional.

Para el caso particular del presente estudio se logró a través de la lectura conocer los componentes de las diferentes temáticas que ellos quisieron trabajar de acuerdo a sus intereses propiciando de esta manera la libertad de decisión asociada a los gustos e intereses particulares.

\section{Referencias}

Camargo, C. (2015). La investigación como estrategia pedagógica en la Guajira, desde una perspectiva de la inclusividad y diversidad como nuevo paradigma para el desarrollo de habilidades, destrezas y competencias. Educación y ciudad, (29), 149 162.

Estados Unidos Mexicanos. Secretaria de educación Pública. (2001). Nuestros nuevos alumnos en secundaria. México.

Figueroa, H., y Carrascal, A. (2016). Potencialización del pensamiento crítico a través de la Hipotemetacomprensión Textual (HMT). Cultura Educación y Sociedad, 7(2), 54-71.

Franco, M. (2010). Estrategias de enseñanza y la comprensión lectora. $R e$ vista Cultura, Educación, Sociedad-CES, 1(1).

Franco, M., Blanco, P., Cortés, O. (2013). Papel de las habilidades metalingüísticas en los procesos de lectura y escritura en la educación superior. Escenarios, 11(2), 82-86.

Fuchs, D., Mock, D., Morgan, P., y Young, C. (2003). Responsiveness-tointervention: Definitions, evidence, and implications for the learning disabilities construct. Learning disabilities research and practice. 18, 157-171.

Gómez, J. (2011). Comprensión lectora y rendimiento escolar: una ruta para mejorar la comunicación. Revista de Investigación en Comunicación y Desarrollo, 2(2). 
Gómez, T., Molano, O., y Rodríguez, S. (2015). La Actividad Lúdica como estrategia Pedagógica para Fortalecer el Aprendizaje de los niños. Universidad de Tolima. Ibagué-Tolima.

Goodman, K. (1982). El proceso de la lectura: consideraciones a través de las lenguas y del desarrollo. En Ferreiro y Gómez Palacio. Nuevas perspectivas sobre los procesos de lectura y escritura. México: Siglo XXI.

Gutiérrez, B., y Pérez, H. (2012). Estrategias de comprensión lectora: enseñanza y evaluación en educación primaria. Revista de Currículum y Formación de Profesorado, vol. 16, núm. 1.

Hernández, R., Fernández, C., y Baptista, P. (2010). Metodología de la investigación. Editorial Mc Graw Hill. México.

Manzano, M. (2000). Impacto del léxico en la lectura de comprensión en los estudiantes de la secundaria técnica $N^{\circ} 1$ en el Estado de Tlaxcala. Tesis. Universidad Autónoma de Tlaxcala: Tlaxcala, México.

Mariño, G. (2010). El diálogo en la educación de jóvenes y adultos. Dos propuestas pedagógicas para implementarlo. En: El taller dialógico / la recuperación de experiencias laborales. Bogotá. OEI.

Marín, F., Niebles, M., Sarmiento, M., y Balvuena, S. (2017). Mediación de las tecnologías de la información en la comprensión lectora para la resolución de problemas aritméticos de enunciado verbal. Revista Espacios Vol. $38\left(N^{o} 20\right)$.
Marr, M., y Gormley, K. (1982). Children's recall of familiar and unfamiliar text. Reading Research Quarterly, 18, 89104.

Mayer, R. (2002). Psicología educativa. Prentice-Hall. Madrid.

Medina, G., y Vargas, N. (2015). La lúdica como estrategia pedagógica para los niños del grado primero. Universidad del Tolima, Ibagué.

Mejía, M., y Manjarrés, M. (2010). Las pedagogías fundadas en la investigación. Búsquedas en la reconfiguración de la educación. Revista Internacional Magisterio 42: 16-26. Colombia.

Ortega, P., Peñuela, D y López, D. (2009). Sujetos y prácticas de la pedagogía crítica. Ediciones El Búho. Bogotá.

Paris, G., Lipson, M., y Wixson, K. (1983). Becoming a strategic reader. Contemporary Educational Psychology, 8, 293-316.

Picardo, O., Balmore, R., y Escobar, J. (2004). Diccionario enciclopédico de ciencias de la educación. San Salvador: El Salvador.

Ramírez, R. (2018). Tendencias emergentes de la gestión de talento humano en las organizaciones. Pp. 101-107.

Restrepo, P., Gutiérrez, M., Caro, N., y Moreno, C. (2015). La lúdica como estrategia pedagógica para fortalecer la convivencia escolar. Lúdica Pedagógica, (21).

Rodríguez, F. (2007). Competencias comunicativas, aprendizaje y enseñanza de las Ciencias Naturales: un enfoque lúdico. Revista electrónica de enseñanza de las ciencias, 6(2), 275298. 
Romero, L., Escorihuela, Z., y Ramos, A.

(2009). La actividad lúdica como estrategia pedagógica en educación inicial.

Revista efdeportes, 14(131). 